CERN-PH-EP/2005-056

December 8, 2005

Submitted to Nucl. Instr. and Meth. Sect. A

\title{
Ion feedback suppression in Time Projection Chambers
}

\author{
F. Sauli ${ }^{*}$ L. Ropelewski and P. Everaerts \\ CERN, CH-1211 Geneva, Switzerland
}

\begin{abstract}
A controlled-voltage Gas Electron Multiplier (GEM) can be used to block the re-injection of positive ions in large volume Time Projection Chambers. With proper choice of geometry, gas filling and external fields, good electron transmission can be obtained at very low GEM voltages; pulsed ion gating is then much easier than with conventional wire grids, requiring hundreds of volts. Gating schemes suited for the TPC detector planned for the International Linear Collider detector are described. The possibility of GEM-based DC-operated ion filters, exploiting the difference in diffusion properties of ions and electrons, is also discussed.
\end{abstract}

Keywords: Ion feedback; Gas Electron Multiplier; Time Projection Chamber; International Linear Collider; Gaseous photon detectors; Ion filter

PACS: 29.40.Gx; 29.40.Cs

\section{Introduction}

Use of a Gas Electron Multiplier (GEM) end-cap detectors for the readout of Time Projection Chambers (TPC) offers numerous advantages, as compared to a conventional Multi-Wire Proportional Chamber readout: very good single-point accuracy and multi-track resolution in projection, due to the narrower pad response function; excellent resolution in the drift direction, resulting from the absence of ion tails in the detected signal; negligible ExB track distortion effects; substantial reduction of ion feedback [1]. Other advantages include robustness (no thin wires) and freedom of shapes, as GEM electrodes can be tailored to the needs.

Motivated by the requirements of the detectors under design for the International Linear Collider (ILC) [2], a large amount of work has been devoted recently to assess the operating performances of GEM-TPC tracking devices, in particular for operation in high magnetic fields [3-10]. A constant charge gain and a point accuracy around $100 \mu \mathrm{m} \mathrm{rms}$ have been demonstrated in prototype detectors operated in high magnetic fields [7].

Throughout this paper, we define fractional ion feedback the ratio of the (positive) charge injected into the drift volume and collected on the drift electrode, to the (negative) electron charge collected on the anode. As will be discussed later, GEM structures are rather efficient in reducing the fractional ion feedback, particularly in

\footnotetext{
*Corresponding author: Tel.: +41(0) 227673670 E-mail: fabio.sauli@ cern.ch (F. Sauli)
} 
presence of high magnetic fields; at 4 tesla, it has been found to be as low as $0.2 \%$ [7]. Despite this small value, compared to the one observed in MWPCs (typically 10-20\%), the operation at even moderate rates can be affected by positive ion accumulations in the drift volume, inducing rate-dependent modifications of the electric field strength and direction with ensuing deformations in the electron drift trajectories, exacerbated by the presence of a high magnetic field [11]. Direct measurements of track distortions in presence of a positive ion charge accumulation in the drift volume, discussed for example in Ref. [12], can be orders of magnitude larger than the desired position accuracy.

A "rule of thumb" for the acceptable feedback comes from the consideration that, with existing electronics, a proportional gain around $10^{4}$ is required for efficient tracking of minimum ionizing particles; a fractional feedback of $10^{-4}$ would then equal the positive ions re-injected in the drift volume, to those released anyhow by primary ionization. Only a detailed calculation taking into account the physics-dependent primary charge release distribution, the geometry of the detector and the acceptable amount of distortions can provide a better quantitative answer.

Ion backflow and feedback processes have been extensively studied in the development of GEM-based gaseous photomultipliers, with the introduction of innovative structures and gating electrodes aimed at reducing ion-induced secondary processes, see [13] and references therein.

\section{Positive ion feedback and suppression}

Charge transport and ion feedback processes in GEM-like structures have been studied by many authors, both theoretically and experimentally [14-21]. For a single GEM, the fraction of ions receding into the drift volume equals approximately the ratio of drift to transfer fields; in multi-GEM detectors, since most ions are generated in the last multiplication stage and are partly collected by the intermediate electrodes, this value is further reduced. For a double-GEM and with a ratio 0.05 between drift and transfer fields, it amounts to around $2 \%$ [15].

The ion feedback ratio is affected by the electrodes geometry, transfer field strength and gas mixture used. In a systematic work of optimization, the authors of Ref. [20] succeeded in reducing this value to $\sim 0.5 \%$, using a triple-GEM with middle electrode having smaller holes. As mentioned, a strong magnetic field permits to reduce further the fractional feedback, to around $0.2 \%$, still however exceeding the target value of $10^{-4}$.

An intermediate grid or wire mesh pulsed with proper timing can be used to stop ions entering the drift volume [22]; "ion gates" have been implemented in the large TPCs used at CERN's Large Electron Positron collider, allowed by the time structure of the beams [23]. Gating seems however not feasible for continuous or long-spill machines.

A convenient gas choice in Time Projection Chambers is a mixture of argonmethane in the proportions 90-10, since it provides fast electron drift at low drift fields, thus requiring a moderate value of the voltage for long drift volumes. In this mixture, the electron drift velocity peaks at around $6 \mathrm{~cm} / \mu \mathrm{s}$ for a field of $150 \mathrm{~V} / \mathrm{cm}$; slowly varying around the peak, the drift velocity is also less sensitive to pressure and temperature variations. The small value of drift field implies also a reduced ion feedback fraction, when entering a higher field region, both for a conventional and for a GEM-based 
readout.

In the quoted mixture, the mobility of $\mathrm{CH}_{4}$ ions (the only species left after charge transfer collisions, due to their lower ionization potential) is, at STP, $\sim 1.9 \mathrm{~cm}^{2} \mathrm{~V}^{-1} \mathrm{~s}^{-1}$ [24]. In a multi-GEM structure, most of the feedback ions are produced in the last step of multiplication (see section 3); with two, $2 \mathrm{~mm}$ thick transfer gaps with $3 \mathrm{kVcm}^{-1}$ applied, they enter the drift region about $70 \mu \mathrm{s}$ after generation and move there (in $150 \mathrm{Vcm}^{-1}$ ) with a velocity of $\sim 290 \mathrm{~cm} \mathrm{~s}^{-1}$.

In the current design, the International Linear Collider (ILC) has $0.95 \mathrm{~ms}$ bunch train spills, spaced $0.2 \mathrm{~s}$ apart; a large TPC surrounds the beam-crossing region, and detects tracks produced in the collisions. Positive ions generated all along a spill make up a layer of charge about $3 \mathrm{~mm}$ thick, slowly receding into the drift volume; at the next spill, the layer will be about $60 \mathrm{~cm}$ away from the end cap. Would the TPC length be equal or shorter, there will be no problem; for longer devices, however, several layers accumulate in the volume and modify direction and strength the field, with consequent track distortions.

A possible way to overcome this problem would be to use higher drift fields, in order to clear up the ions in the time between collisions; this requires however uncomfortably large values of voltage. A conventional pulsed gating could be used, adding a wire mesh above the end-cap detector; however, aside from the large mechanical and electrical complication of implementing such a scheme, the discrete structure of the mesh, with wires a few mm apart, is bound to introduce field distortions and ExB effects, partly negating the advantages of a GEM readout. An alternative solution, exploiting the transmission properties of multi-GEM structures is proposed here, after a short reminder on electron and ion flow properties through GEM foils.

\section{GEM charge transmission properties}

Charge transmission through GEM electrodes depends on hole's pattern, applied fields and gas mixture; for multiple structures, the behavior becomes rather complex, as discussed in detail by many authors $[7,15,17,20,21]$. To help the reader, a simplified picture of the charge transfer processes is presented here.

Two basic configurations are considered: electron transport with multiplication from low to high fields, corresponding to the first GEM in a cascade, and from high to high fields, as for the following electrodes in a multiple structure. Fig. 1 shows schematically the charge flow for electrons and ions in the two cases; numbers close to the arrows give approximate estimates for the relative sharing of charges. The values, deduced from Ref. [15], correspond to the operation of a standard GEM, with 70 $\mu \mathrm{m}$ holes at $140 \mu \mathrm{m}$ pitch, in $\mathrm{A}-\mathrm{CO}_{2} 70-30$ at STP, at a moderate voltage $(350 \mathrm{~V})$. Due to the relatively low transfer field, $3 \mathrm{kV} / \mathrm{cm}$, recommended to prevent discharge propagation [25], about $60 \%$ of the electrons in the avalanche are lost to the lower side of GEM. In the example, the real gain of the foil is around 50, while the effective gain, deduced from the electron charge transferred, is $~ 20$ (left side of the diagrams). When collected from a high field region, a fraction of electrons, around $20 \%$, is also lost to the upper GEM electrode.

For positive ions (center and right), two sets of diagrams are shown, corresponding to charges produced in the avalanche, and to those receding from the 
transfer gap, being generated by avalanches in the following foil.

Combining the data for a triple-GEM structure, beginning with a low drift field, one can infer from the diagrams the total effective gain to be $\sim 5000$ (20x16x16). Starting the avalanche process from one electron released in the drift region, $\sim 2$ ions are fed into the drift field from GEM1, 12 from GEM2 and 110 from GEM3. In this example, the fractional ion feedback is about $2.5 \%(123 / 5000)$, in reasonable agreement with the measurements done in similar conditions [15].

A way to eliminate feedback is to introduce a supplementary intermediate drift space, separated by a wire mesh from the main volume of the TPC; this electrode can then be gated close, offsetting the voltage between pairs of adjacent wires, as done in conventional TPCs. Choosing for this region a field of $300 \mathrm{~V} / \mathrm{cm}$, double of the one in the drift to ensure good electron transmission [26], a $6 \mathrm{~mm}$ gap is sufficient to delay the ions by about $1 \mathrm{~ms}$, as required by the ILC operation; their injection in the drift volume can then be inhibited pulse-closing the gate, after the delay, for a time equal to the spill length.

An attractive possibility is to use as gating electrode one of the GEM foils in a multiple structure, with a low field in the first transfer gap, a rather unusual configuration. We have therefore measured electron transmission into moderate and low fields, compared to the standard settings, for a wider range of gases and geometry. The measurements were done with a triple-GEM detector mounting three $10 \times 10 \mathrm{~cm}^{2}$ foils, irradiating the detector with a $9 \mathrm{keV} \mathrm{X}$-ray beam from a generator, and recording the signal pulse height on the anode. The second and third GEM (70 $\mu \mathrm{m}$ holes at $140 \mu \mathrm{m}$ pitch) were operated in the standard, high gain configuration, while varying the transfer field and voltage of the first electrode. In what follows, as only the fields above and below the first GEM in the cascade were varied, they are named simply drift and transfer fields.

To avoid normalization errors, measurements were made with direct detection of pulses, as against the integral current method used in previous work. With the described geometry, the detected pulse height spectrum has two components, corresponding to conversions in the upper drift volume and in the transfer region between first and second GEM; the second contribution can be easily resolved inverting the drift field, and subtracted from the first. Comparison of the two spectra provides an absolute estimate of the electron fraction transferred from the first foil; this quantity is referred to as electron transmission ${ }^{1}$. An example is given in Fig. 2, showing total, inverted drift and subtracted spectra for the case of very low first GEM voltage (well before charge multiplication sets in). The relative counting rates of the two components correspond to the gap thickness (6.5 and $2 \mathrm{~mm}$ ), taking into account the absorption losses in the GEM foil, and the ratio between the peaks provides the value of fractional electron transmission through the first electrode, around 0.7 for the example given. Due to transmission losses, the energy resolution is degraded (from 17\% to $\sim 30 \%$ FWHM for the $9 \mathrm{keV}$ line), probably good enough for tracking. Increasing the first GEM voltage above its multiplication threshold, one gets higher signals with better resolution, $25 \%$ FWHM, still however worse than for a standard operation, demonstrating the effect of losses due to the low transfer field value (Fig. 3).

\footnotetext{
${ }^{1}$ The term "transmission" is used improperly at high GEM voltages, where charge multiplication occurs.
} 
Fig. 4 provides the measured electron transmission for a standard GEM (70 $\mu \mathrm{m}$ holes at $140 \mu \mathrm{m}$ pitch), in Ar- $\mathrm{CO}_{2} 70-30$ and transfer fields of 150,300 and $3000 \mathrm{Vcm}^{-1}$. The transmission is unexpectedly large at very low GEM voltage and transfer field: about $30 \%$ for $\mathrm{V}_{\mathrm{GEM}}=10 \mathrm{~V}, \mathrm{E}_{\mathrm{D}}=150 \mathrm{Vcm}^{-1}$ and $\mathrm{E}_{\mathrm{T}}=300 \mathrm{Vcm}^{-1}$. At higher GEM voltages, one enters the multiplication region, where the detected signal is the product of transmission and effective gain, and the two contributions cannot be resolved.

The electron transmission in the low voltage region depends on the gas mixture, as (Fig. 5); it reaches $50 \%$ for equal fractions of argon and $\mathrm{CO}_{2}$. As discussed in the next section, this is likely due to a reduction in the electrons' transverse diffusion in the quencher-rich mixtures. The observation is confirmed using as first electrode a foil with larger holes (100 instead of $70 \mu \mathrm{m}$, at $140 \mu \mathrm{m}$ pitch): the low voltage transmission increases even further, to about $70 \%$ (Fig. 6).

Operating the first GEM foil in a cascade at very low voltage is a very attractive proposition; while in this case the foil does not contribute to amplification, ion gating can be achieved with only ten volts of reverse pulse, as against the several hundred required using a wire mesh or operating the GEM electrode in the high gain region. It should be note however that transmission losses in the first GEM may affect the energy resolution of a TPC and deteriorate its particle identification properties.

Detailed studies of gain and discharge probability in multi-GEM structures have demonstrated that, in absence of heavily ionizing background, the required gain for a TPC of $\sim 10^{4}$ can be reached with a double-GEM [25]; in case of need, a third multiplier could be added. Alternatively, and at the cost of requiring a higher voltage pulse for gating, the first foil can be used also for multiplication. As seen in the figures, and because of the small value of the first transfer field, the effective gain is reduced as compared to normal operation; at $350 \mathrm{~V}$, it adds a factor of ten to the gain of the cascade.

It should be mentioned that the standard design of large area GEM foils, with thin and long sectors independently powered to limit the energy in case of a discharge [27], has very conveniently the characteristics of a multiple transmission line; pulsed strips can be individually $\mathrm{AC}$ terminated at the far end on their characteristic impedance, suppressing reflections and therefore reducing noise pickup and ringing inevitable in a pulsed gating.

In Fig. 7, a schematic timing diagram for the ion backflow is shown for a triple GEM detector, with the first foil in the cascade placed six mm from the second and with a transfer field of $300 \mathrm{Vcm}^{-1}$. Adopting a lower value $\left(150 \mathrm{Vcm}^{-1}\right)$, this distance can be reduced to $3 \mathrm{~mm}$; however, as seen in Fig. 4, the tolerance on the GEM voltage ensuring good transmission may be more critical and raise concerns on the uniformity of response over large areas. For different gas mixtures, the gap thickness and/or the transfer field value will have of course to be adjusted to the species and mobility of the drifting ions.

\section{Discussion of the results}

Electrons released by ionization in the drift volume move towards and through the multi-GEM structure following the field lines. In absence of diffusion, the charge sharing could be deduced simply from a field line count. Because however of the smearing due to diffusion, and in particular of its transverse component, perpendicular to the field, a fraction of electrons can be lost to the intermediate electrodes, before reaching the anode, 
even if there are no connecting lines. The charge spread is particularly large during the avalanche development within the narrow holes. As already shown in Ref. [21], the transmitted fraction of electrons depends on gas and GEM geometry, with a general trend to increase with the increase in the quencher amount and of the GEM holes' diameter. This is demonstrated in the present work to hold also for low transfer fields and GEM voltages.

Using the program MAGBOLTZ [28] we have computed the drift properties of electrons in several gases. Fig. 8 shows the transverse diffusion, for one $\mathrm{cm}$ of drift, as a function of field for several argon- $\mathrm{CO}_{2}$ mixtures at STP, and no magnetic field (full curves); dashed curves correspond to $\mathrm{Ar}-\mathrm{CH}_{4}$ (90-10) and $\mathrm{Ar}-\mathrm{CH}_{4}-\mathrm{CO}_{2}$ in the proportions 93-5-2, in a magnetic field of 4 tesla parallel to the electric field. The last mixture, that preserves many of the favorable characteristics of the standard argon-methane mixture, is non-flammable and a likely candidate for use in large TPCs $[2,7]^{2}$. One can see that in the region corresponding to a low voltage operation of GEM (few hundred $\mathrm{Vcm}^{-1}$ to a $\mathrm{kVcm}^{-1}$ ), the values of the transverse diffusion for $\mathrm{A}-\mathrm{CO}_{2} 70-30$, at $\mathrm{H}=0$, are comparable to those of the Ar- $\mathrm{CH}_{4}-\mathrm{CO}_{2}$ mixture at 4 tesla, suggesting a similarity in the transmission characteristics. It should be noted that the authors of Ref. [7], operating a standard tripleGEM detector, reported a two-fold increase of the anode current from zero to five tesla; exploiting the results of a GARFIELD simulation [29], this was explained as being due to an increase of the extraction drift lines ${ }^{3}$. In our understanding, the increase is due instead to the reduction of transverse diffusion losses; the reduction in positive ion feedback (also by a factor of two) is then in fact due to the increase in the electron signal at the anode. In view of the dependence of electron transmission from the gas properties, this is of course a crucial point, and will require experimental verification.

In the described scheme, electrons from tracks produced during a spill traverse the gap between gating and first multiplying GEM, while the positive charge builds-up in the same region. Due to the narrowness of the gap, distortions are expected to be small or negligible; indeed, in the expected ILC running conditions, the positive charge density in the layer is smaller than for a standard thin-gap triple-GEM detector at high fluxes. Operational experience at high rates has shown only a small deterioration of position accuracy, from $\sim 60 \mu \mathrm{m}$ at low rates to $\sim 70 \mu \mathrm{m}$ at a particle flux of $\sim 10^{4} \mathrm{~mm}^{-2} \mathrm{~s}^{-1}$ [30]; however, the worsening could be entirely due to signal pileups and software reconstruction difficulties. The measured gain uniformity up to very high fluxes (above $10^{6} \mathrm{~mm}^{-2} \mathrm{~s}^{-1}$ ) [31] demonstrates the absence of recombination or space-charge related effects. Further work is however required on these points.

Short of waiting the beginning of ILC operation, further evidence for the soundness of the proposed ion suppression scheme could be obtained with a dedicated experiment, using a beam with a time structure close to the one foreseen for the collider. The spill could be emulated with a pulsed X-ray tube, generating $1 \mathrm{~ms}$ bursts at $200 \mathrm{~ms}$ intervals. Alternatively, one could use a continuous generator with a disk collimator, rotating at 300 turns/minute with a slit width corresponding to $1 / 200$ of the circumference. In both cases, the time zero reference could be obtained with a separate

\footnotetext{
2 In the ternary mixture, due to charge transfer collisions, only ions with the lowest ionization potential $\left(\mathrm{CO}_{2}{ }^{+}\right)$are left drifting; their lower mobility $\left(\sim 1 \mathrm{~cm}^{2} \mathrm{~V}^{-1} \mathrm{~s}^{-1}\right)$ has to be taken into account for gating.

3 In the simulation, using the Runge-Kutta option, the transverse diffusion is neglected.
} 
fast detector in line with the beam. Track distortions induced by ions, with and without gating, could be measured with the method described in Ref. [12]: the space coordinates of a collimated X-ray source $\left(5.9 \mathrm{keV}\right.$ from $\left.{ }^{55} \mathrm{Fe}\right)$ are measured, in presence of the ion cloud produced by the generator, as a function of distance and charge density.

\section{Future developments: the "ion filter"}

A thorough understanding of the drift and diffusion properties of ions and electrons trough the GEM foils could lead to an improved "ion filter", a DC-operated structure with the property to transmit electrons in one direction whilst blocking most ions

Fig. 9 shows, as a function of field, the computed transverse diffusion for one $\mathrm{cm}$ drift in pure methane at STP for electrons (full line) and ions (dashed line), For ions, and up to very high values of field (tens of $\mathrm{kVcm}^{-1}$ ) the space diffusion is symmetric, independent from magnetic field and type of ion [32, 33]. At the common values of transfer field between GEMs $\left(3\right.$ to $\left.5 \mathrm{kVcm}^{-1}\right)$, the electron cloud emerging from a hole spreads laterally almost an order of magnitude more than ions; this difference could be exploited with suitable detector geometry to decrease the ion feedback. Mounting two GEM foils at a distance comparable with the hole's pitch, and offset by half of the pitch, most field lines emerging from one hole terminate on the facing GEM electrode (Fig. $10)^{4}$. One expects that a good fraction of the electrons would still make their way through the structure, thanks to the transverse spread, while ions, almost strictly following field lines, would be mostly collected by the facing electrode. This should result in a substantial reduction in the fractional ion feedback; in presence of a strong magnetic field, the filtering can be very effective, as demonstrated in conventional TPCs [34], possibly enough to avoid the need of pulsed gating in TPC devices. The scheme could also be useful to reduce the ion feedback and photocathode degradation problems encountered in the development of photon-sensitive GEM detectors, and operated in pure methane or $\mathrm{CF}_{4}$ [35]. In this case, electron transmission losses would play a small role, in view of the exponential charge distribution for single electron avalanches.

Due to the requirement for the gap be comparable to the hole's pitch, such geometry is difficult to realize with standard GEM foils; it is easier to implement using larger holes and pitch, using the so-called "Thick-GEM" developed by the authors of Ref. [36].To test the principle, we have assembled in a detector two small-size Thick-GEM plates $^{5}$, with two mm transfer gap; the multiplier have a special design, intended to avoid edge discharges, with $300 \mu \mathrm{m}$ diameter holes in the insulator, surrounded by a $100 \mu \mathrm{m}$ wide metal-free rim, and $800 \mu \mathrm{m}$ pitch in a triangular pattern. One GEM could be moved along the direction of a row of holes with a gas-tight external micrometer. To enhance the transverse electron diffusion, obliterating the opacity for electrons of the GEM pair when misaligned, we operated the detector in an argon- $\mathrm{CO}_{2}$ 90-10; at a transfer field around 3 $\mathrm{kVcm}^{-1}$ the diffusion in this gas is close to the one in pure methane (see Figs. 8 and 9). With $1050 \mathrm{~V}$ applied to each GEM, detector was operated at effective gains around 4000; drift and transfer fields were $150 \mathrm{Vcm}^{-1}$ and $3 \mathrm{kVcm}^{-1}$ respectively, a standard choice for

\footnotetext{
4 Assembled from a single cell field map computed by S. Kappler.

${ }^{5}$ Courtesy of A. Breskin and R. Chechik, Weizmann Institute of Sciences, Rehovot.
} 
TPC-like detectors. Pulse height on the anode, and currents on both anode and drift electrode were measured irradiating the detector with a $9 \mathrm{keV} X$-ray beam about one $\mathrm{cm}^{2}$ in section.

Fig. 11 shows the measured anode and drift currents under continuous irradiation, as a function of the sliding GEM micrometer reading. While the electron current is modestly affected, the ion current, measured on the drift electrode, varies considerably; the fractional ion feedback decreases from 10 to $6 \%{ }^{6}$. The sharp shape of the drift current around the minimum is suggestive of an insufficient "opacity", obtained sliding the foil along a row of holes; this could be increased by a diagonal scan, facing the holes to the center of the triangular pattern, or using GEMs with a larger pitch. Pulse height spectra recorded on the anode in the peak and valley of the scan, compared in Fig. 12, show a small variation in the average but no deterioration in resolution, demonstrating the dispersive role of transverse diffusion for electrons.

The results described above demonstrate the effect of a controlled hole's misalignment on drifting charges; the observed ion feedback reduction is however too small to be useful in practice. A detailed study of GEMs' geometry and operating conditions is required to ascertain if the scheme can lead to an effective "DC ion gate". The simplest solution would probably be to design foils with different holes' patterns, optimized to provide maximum average opacity when assembled in pairs, independently from their exact position. The previously quoted observation of reduced ion feedback using one foil with different pitch [20] supports this possibility. The optimization would greatly profit from a detailed simulation study, taking into account the effect of geometry and transverse diffusion.

\footnotetext{
${ }^{6}$ It should be noted that in all works of the authors of Ref. 35, aimed at developing gaseous photocathodes, the "ion backflow" corresponds to the current collected by the upper GEM surface and not the one receding into the drift volume.
} 


\section{References}

[1] F. Sauli, Nucl. Instr. and Meth. A505(2003)195.

[2] T. Behnke, S. Bertolucci, R.D. Heuer, R. Settles, TESLA TDR, DESY-01-011 (2001).

[3] R.K. Carnegie, M.S. Dixit, J. Dubeau, D. Karlen, J.-P. Martin, H. Mes, K. Sachs, Nucl. Instr. and Meth. A538(2005)372.

[4] D. Karlen, P. Poffenberger, G. Rosembaum, Nucl. Instr. and Meth. A555(2005)80.

[5] S. Roth, Nucl. Instr. and Meth. A535(2004)330.

[6] S. Roth, Nucl. Instr. and Meth. A518(2004)103.

[7] M. Killenberg, S. Lotze, J. Mnich, A. Münnich, S. Roth, F. Sefklow, M. Tonutti, M. Weber, P. Wienemann, Nucl. Instr. and Meth. A530(2004)251.

[8] S. Kappler, J. Kaminski, B. Ledermann, T. Müller, L. Ropelewski, F. Sauli, IEEE Trans. Nucl. Sci. NS-51(2004)1524.

[9] S. Kappler, F. Bieser, J. Kaminski, B. Ledermann, T. Muller, M.T.Ronan, L.

Ropelewski, F. Sauli, R. Settles, IEEE Trans. Nucl. Sci. NS-51 (2004)1039.

[10] J. Kaminski, M. Ball, F. Bieser, M. Janssen, S. Kappler, B. Ledermann, T. Müller, M. Ronan, P. Wienemann, Nucl. Instr. and Meth. A535(2004)201.

[11] W. Blum and L. Rolandi, Particle detection with Drift Chambers. Springer-Verlag, Berlin (1993).

[12] D. Friedrich, G. Melchart, B. Sadoulet, F. Sauli, Nucl. Instr. and Meth. 158(1979)81.

[13] A. Breskin, D. Mormann, A. Lyashenko, R. Chechik, F.D. Amaro, M. Maia, J.F.C.A. Veloso, J.M.F. dos Santos, Nucl. Instr. and Meth. A553(2005)46.

[14] J. Benlloch, A. Bressan, M. Capeáns, M. Gruwé, M. Hoch, J.C. Labbé, A. Placci, L. Ropelewski, F. Sauli, Nucl. Instr. and Meth. A419(1998)410.

[15] S. Bachmann, L. Bressan, L. Ropelewski, F. Sauli, A. Sharma, D. Mörmann, Nucl. Instr. and Meth. A438(1999)376.

[16] C. Richter, A. Breskin, R. Chechik, D. Mörmann, G. Garty, A. Sharma, Nucl. Instr. and Meth. A478(2002)538.

[17] A. Buzulutskov, Nucl. Instr. and Meth. A494(2002)148.

[18] V. Tikhonov and R. Veenhof, Nucl. Instr. and Meth. A 478(2002)452.

[19] W. Bonivento, A. Cardini, D. Pinci, IEEE Trans. Nucl. Sci. NS-49(2002)1638.

[20] A. Bondar, A. Buzulutskov, L. Shekhtman, A. Vasiljev, Nucl. Instr. and Meth. A496(2003)325.

[21] F. Sauli, S. Kappler, L. Ropelewski, IEEE Trans. Nucl. Sci. 50(2003)803.

[22] D.A. Bryman, M. Leitch, I. Navon, T. Numao, P. Schlatter, M.S. Dixit, H. Mes,

C.K. Hargrove, J.A. Macdonald, R. Skegg., J. Spuller, R.A. Burham, M. Hasinoff, J.-M. Poutissou, M. Blecher, K. Gotow, A.L. Carter, Nucl. Instr. and Meth. A234(1985)42.

[23] S.R. Amendolia, M. Binder, W. Blum, e. al, Nucl. Instr. and Meth. A252(1986)403.

[24] G. Schultz, G. Charpak, F. Sauli, Revue de Physique Appliquée 12(1977)67.

[25] S. Bachmann, A. Bressan, M. Capeàns, M. Deutel, S. Kappler, B. Ketzer, A.

Polouektov, L. Ropelewski, F. Sauli, E. Schulte, L. Shekhtman, A. Sokolov, Nucl. Instr. and Meth. A 479(2002)294.

[26] O. Bunemann, T.E. Cranshaw, J.A. Harvey, Canadian J. of Res. 27A(1949)191. 
[27] C. Altunbas, M. Capéans, K. Dehmelt, J. Ehlers, J. Friedrich, I. Konorov, A. Gandi, S. Kappler, B. Ketzer, R.D. Oliveira, S. Paul, A. Placci, L. Ropelewski, F. Sauli, F.

Simon, M. vanStenis, Nucl. Instr. and Meth. A490(2002)177.

[28] S. Biagi, Nucl. Instr. and Meth. A421(1999)234.

[29] R. Veenhof, Nucl. Instum. and Meth. A419(1998)726.

[30] B. Ketzer, Q. Weitzel, S. Paul, F. Sauli, L. Ropelewski, Nucl. Instr. and Meth. A535(2004)314.

[31] J. Benlloch, A. Bressan, C. Büttner, M. Capeáns, M. Gruwé, M. Hoch, J.C. Labbé, A. Placci, L. Ropelewski, F. Sauli, A. Sharma, R. Veenhof, IEEE Trans. Nucl. Sci. NS45(1998)234.

[32] E.W. McDaniel and E.A. Mason, The mobility and diffusion of ions in gases.

Wiley \& Sons, New York (1973).

[33] F. Sauli, Principles of operation of multiwire proportional and drift chambers, CERN 77-09 (1977).

[34] S.R. Amendolia, W. Blum, R. Benetta, M. Cherney, F. Fidecaro, J.P. Froberger, B. Hubbard, R.C. Jared, I. Lehraus, F. Liello, P.S. Marocchesi, R. Mattheweson, J. May, T.C. Meyer, E. Milotti, F. Nanni, A. Peisert, M.J. Price, F. Ragusa, J. Richstein, R. Richter, L. Rolandi, W.D. Schlatter, J. Sedgbeer, R. Settles, U. Strierlin, M. Takashima, W. Tejessy, G. Tromba, W. Witzeling, S.L. Wu, W. Wu, Nucl. Instr. and Meth. A234(1985)47.

[35] D. Mörmann, A. Breskin, R. Chechik, D. Bloch, Nucl. Instr. and Meth. A516(2004)315.

[36] R. Chechik, A. Breskin, C. Shalem, Nucl. Instr. and Meth. A553(2005)35. 


\section{Figure Captions}

Fig. 1: Schematic representation of the fractional charge transfer for electrons (left) and ions (center and right) in two GEM configurations: low-to-high (top) and high-to-high electric field (bottom).

Fig. 2: Pulse height spectra recorded in a triple-GEM detector for $9 \mathrm{keV} \mathrm{X}$-rays at low transfer field and GEM voltage $\left(300 \mathrm{Vcm}^{-1}\right.$ and $10 \mathrm{~V}$, respectively). The higher peak corresponds to the sum of conversions in the drift and between the first and second GEM foil; the contribution of conversions in the transfer region can be measured inverting the drift field, and subtracted.

Fig. 3: Pulse height spectra recorded in a triple-GEM detector for $9 \mathrm{keV} \mathrm{X-rays} \mathrm{at} \mathrm{low}$ transfer field and high GEM voltage $\left(300 \mathrm{Vcm}^{-1}\right.$ and $400 \mathrm{~V}$, respectively).

Fig. 4: Electron transmission of a standard GEM foil, measured in the pulse mode, for standard high $\left(3 \mathrm{kVcm}^{-1}\right)$ and low transfer fields $\left(150\right.$ and $\left.300 \mathrm{Vcm}^{-1}\right)$, as a function of GEM voltage. Drift field: $150 \mathrm{Vcm}^{-1}$, gas filling $\mathrm{A}-\mathrm{CO}_{2} 70-30$ at STP.

Fig. 5: Electron transmission of a standard GEM, for low transfer field $\left(300 \mathrm{Vcm}^{-1}\right)$, as a function of GEM voltage and for three A-CO $\mathrm{CO}_{2}$ mixtures: 90-10, 70-30 and 50-50.

Fig. 6: Comparison of electron transmission for two GEM foils: standard (70 $\mu \mathrm{m}$ holes at $140 \mu \mathrm{m}$ pitch) and large (100 $\mu \mathrm{m}$ holes at $140 \mu \mathrm{m}$ pitch). Gas filling: $\mathrm{A}-\mathrm{CO}_{2} 70-30$.

Fig. 7: Timing diagram of the feedback ion flow in a triple-GEM structure, with the first foil, facing the drift volume, at larger distance and with low transfer field $\left(300 \mathrm{Vcm}^{-1}\right)$. Ions produced mostly in the last multiplier (GEM3) during the $950 \mu$ s spill flow towards the gating electrode (GEM1), that they begin reaching $\sim 1 \mathrm{~ms}$ later. A blocking gate can be applied at this time to prevent them entering the drift volume.

Fig. 8: Computed transverse diffusion, for one $\mathrm{cm}$ of drift, as a function of field in several A- $\mathrm{CO}_{2}$ mixtures and $\mathrm{H}=0$ (full lines). The dashed lines show the same quantity for two methane-containing gases, $\mathrm{A}-\mathrm{CH}_{4}$ (90-10) and $\mathrm{A}-\mathrm{CH}_{4}-\mathrm{CO}_{2}$ (90-5-5) computed for a magnetic field of 4 tesla parallel to the electric field.

Fig. 9: Electron transverse diffusion in pure $\mathrm{CH}_{4}$ (full line), and ion diffusion (dashed line), as a function of electric field.

Fig. 10: Schematics of a possible double-GEM "DC ion gate", exploiting the large difference in transverse diffusion between electrons and ions. External fields: $150 \mathrm{Vcm}^{-1}$; central field: $2.5 \mathrm{kVcm}^{-1}$, GEM voltage: $100 \mathrm{~V}$.

Fig. 11: Modulation of the drift and anode currents, and of their ratio, as a function of the relative position of the holes' rows in two facing Thick-GEM plates. The Fractional Ion Feedback (FIF) is reduced by $\sim 40 \%$ for misaligned holes.

Fig. 12: Anode pulse height spectra recorded on the minimum and maximum of the previous plot. 


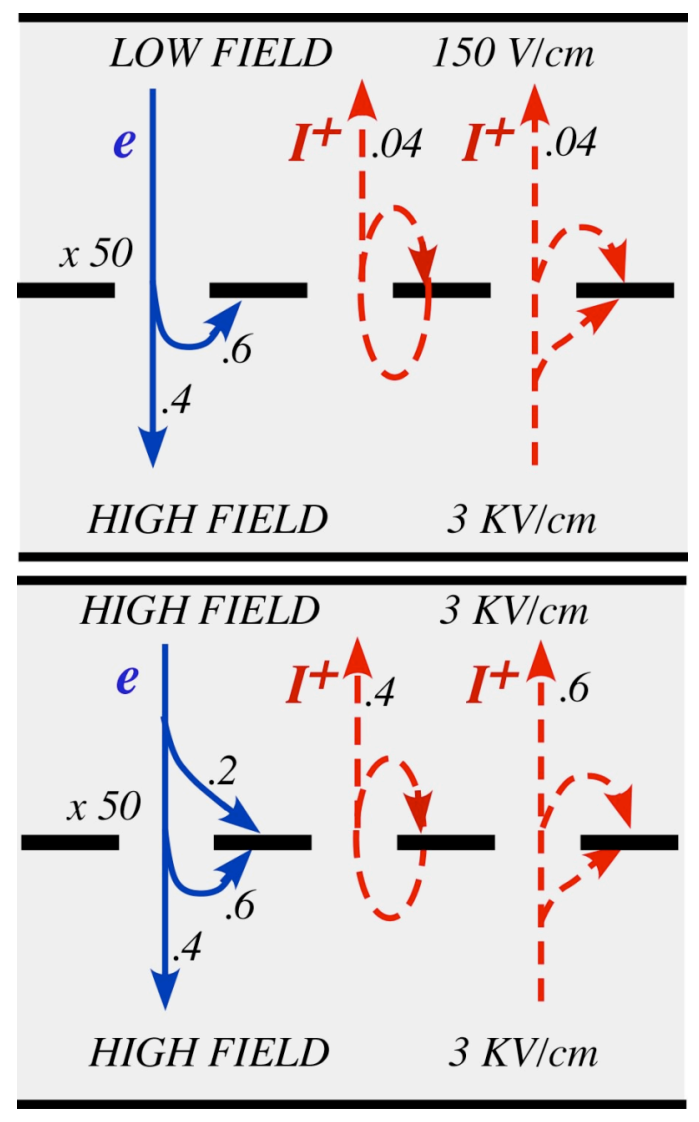

Fig. 1

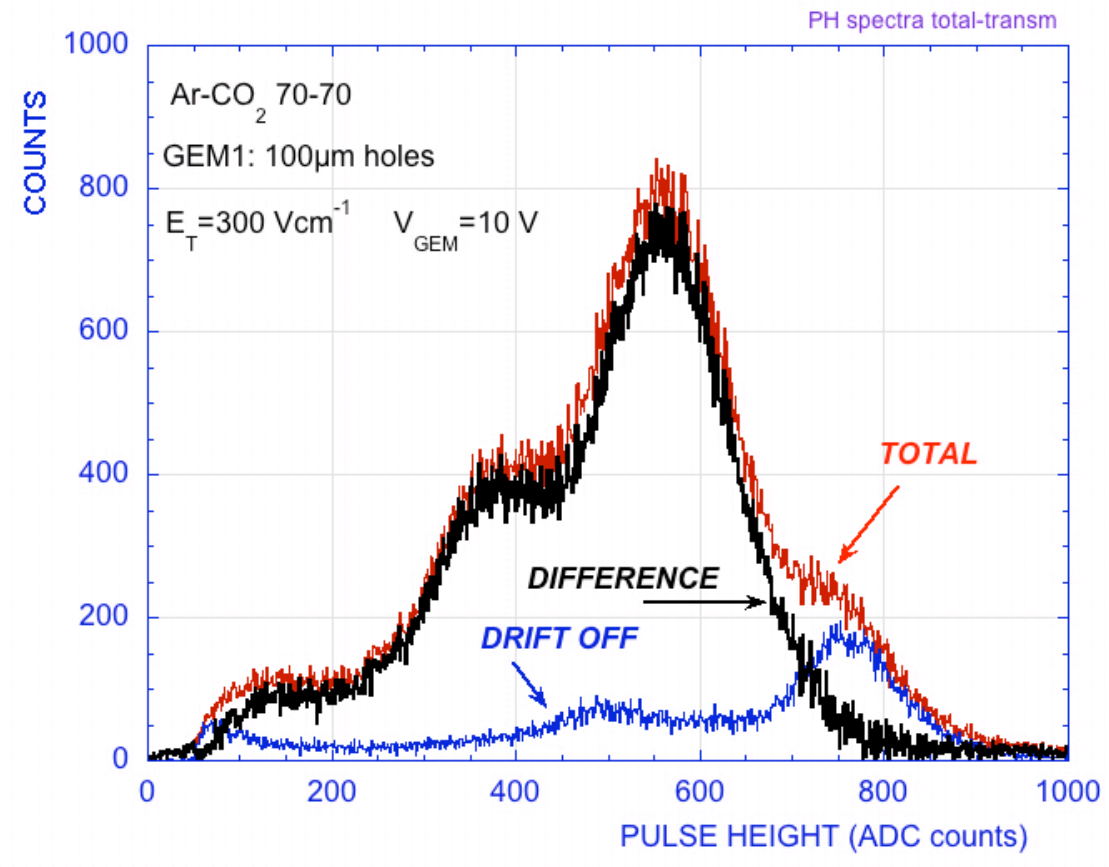

Fig. 2 


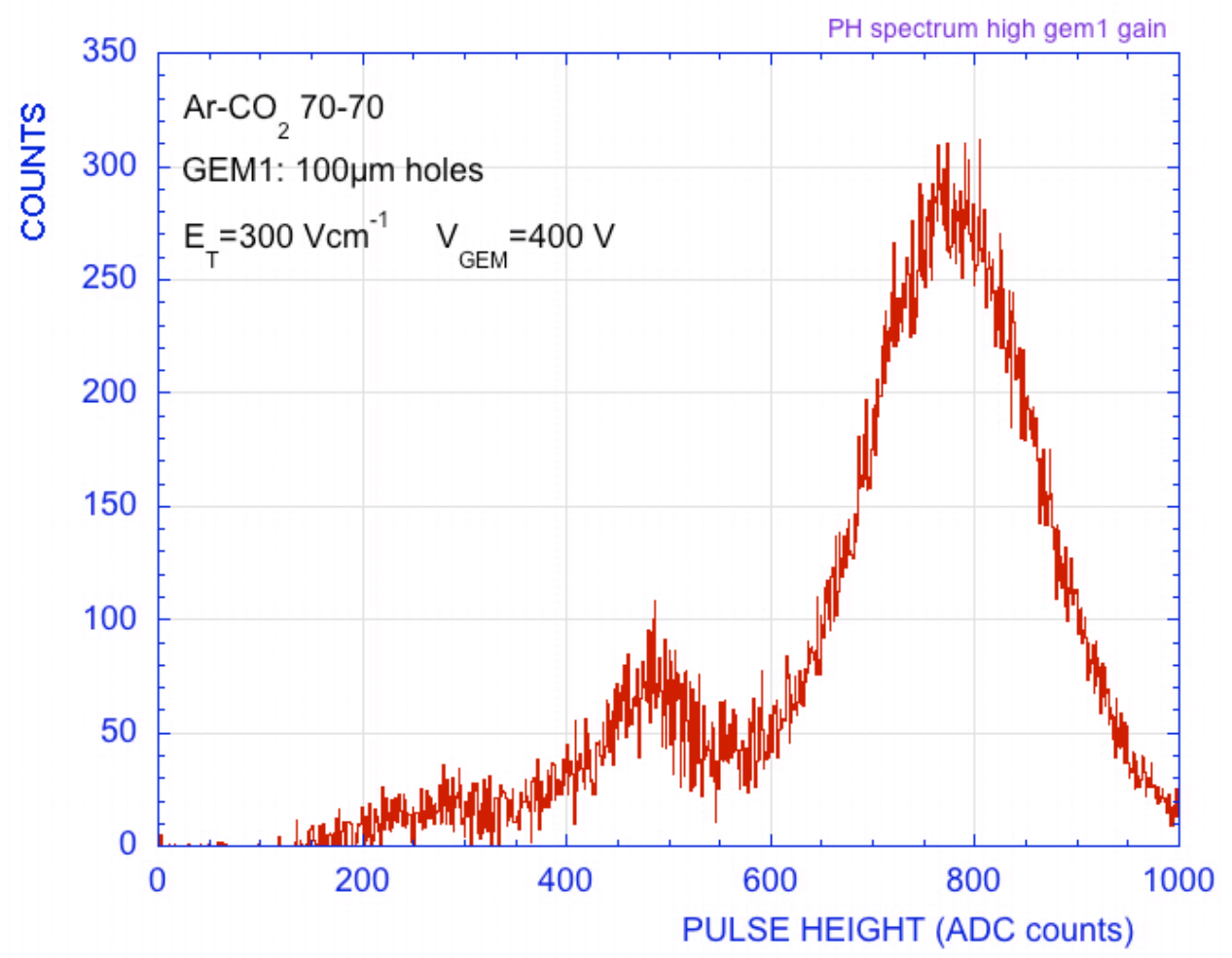

Fig. 3

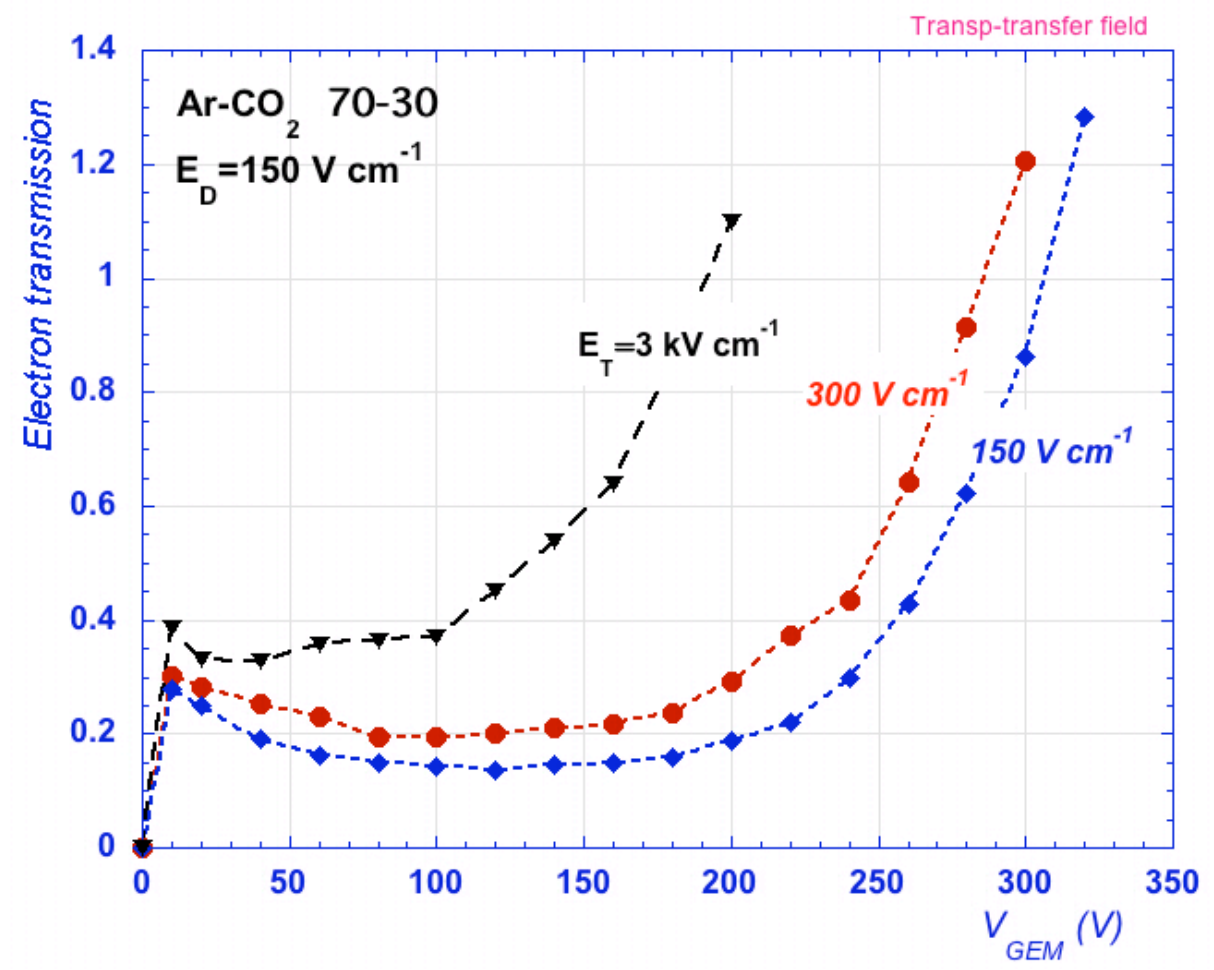

Fig. 4 


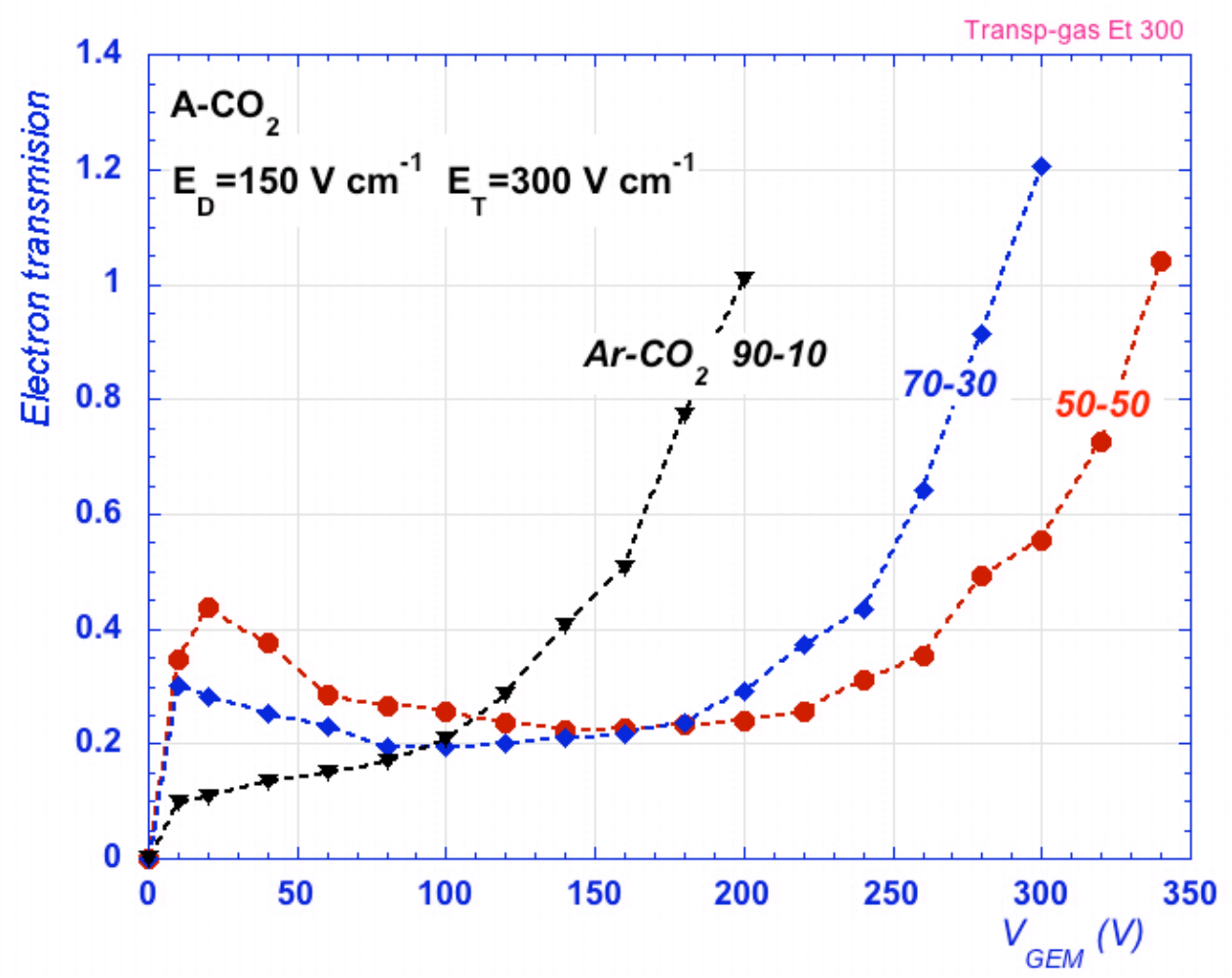

Fig. 5

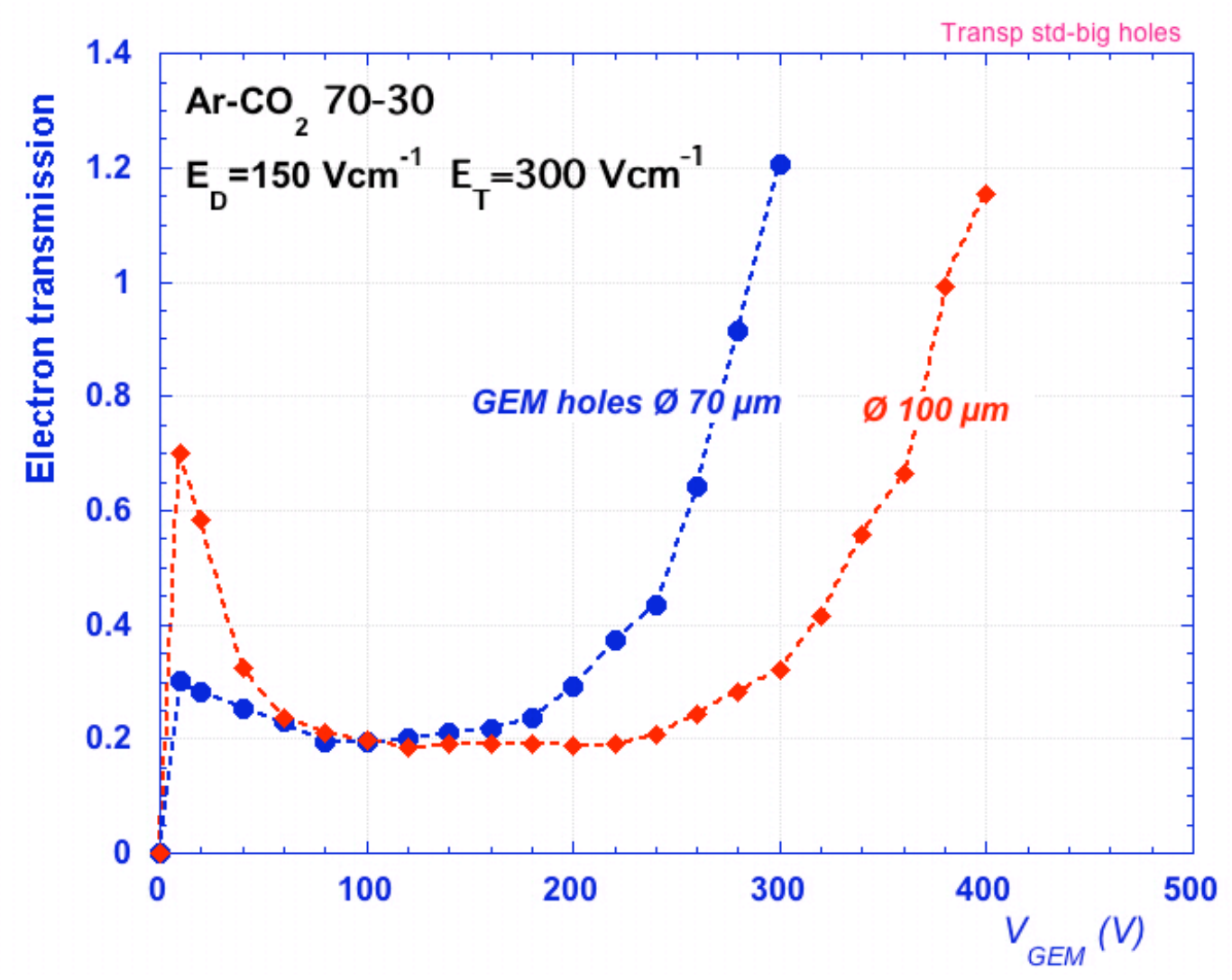

Fig. 6 


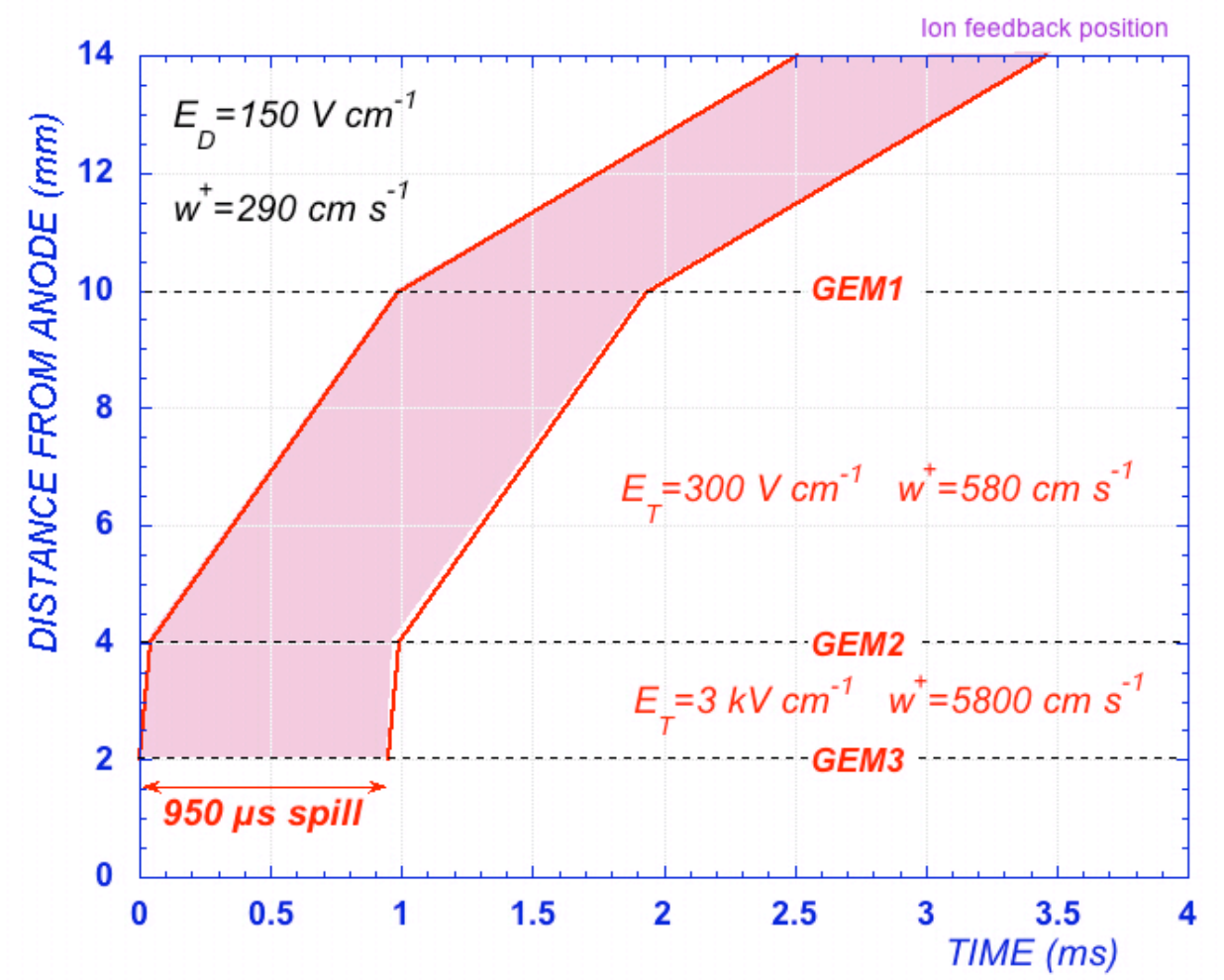

Fig. 7

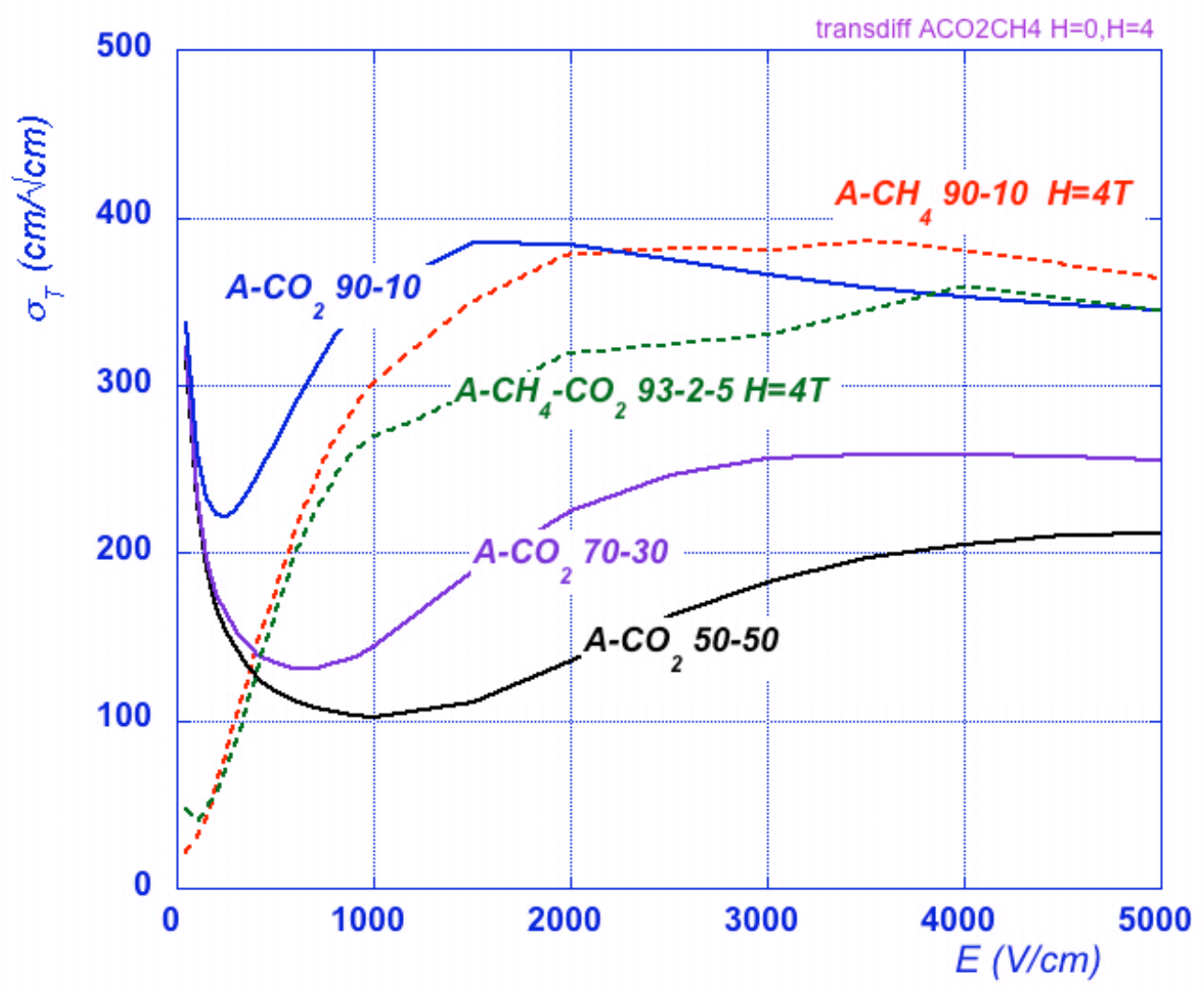

Fig. 8 


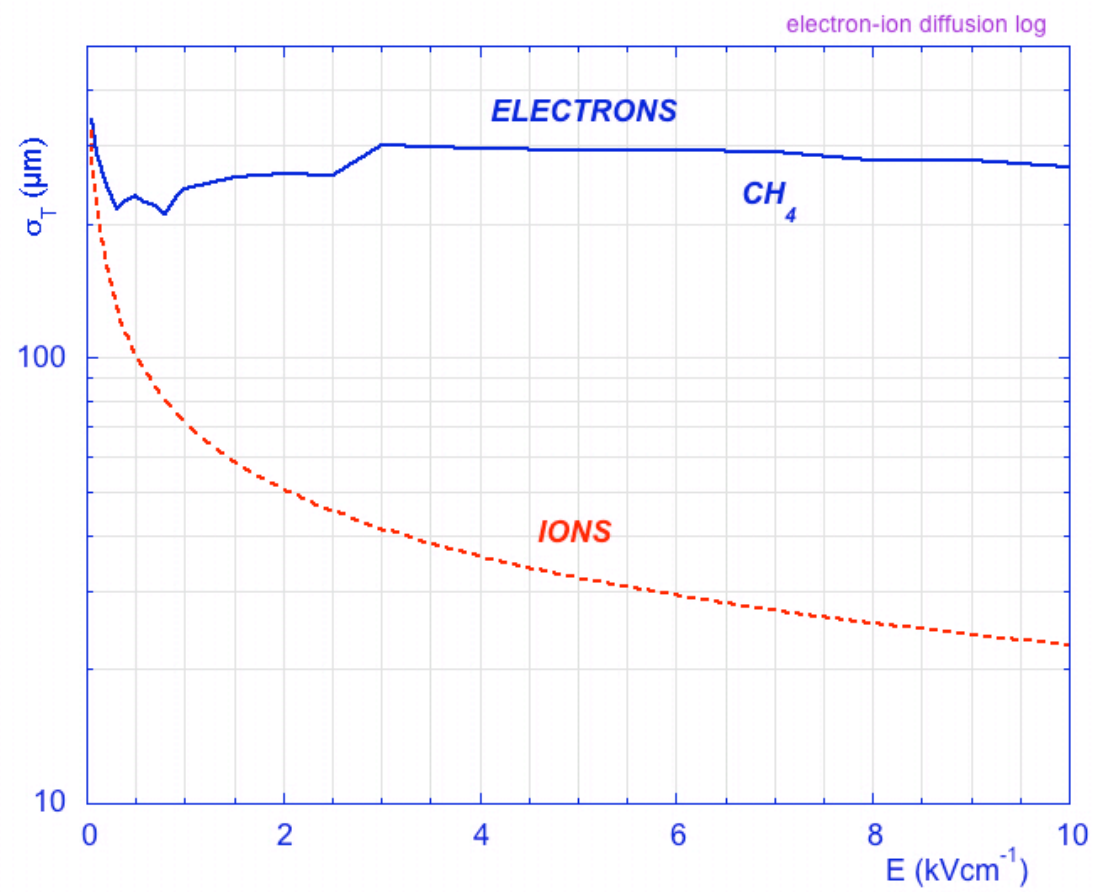

Fig. 9

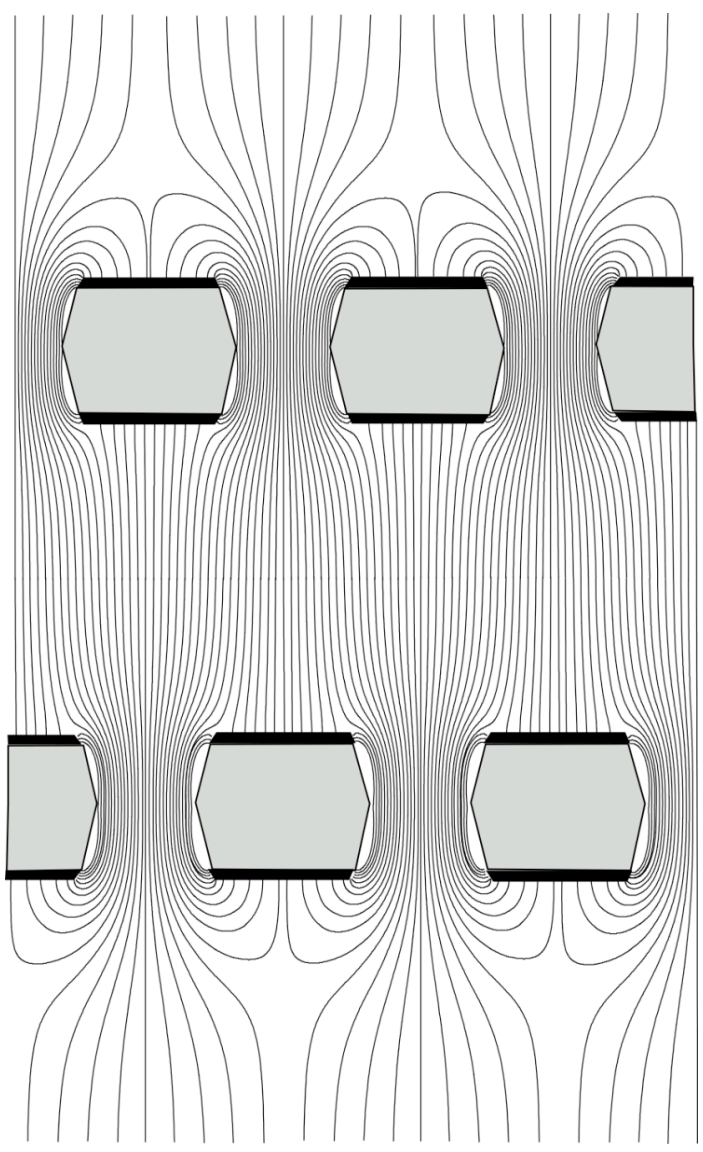

Fig. 10 


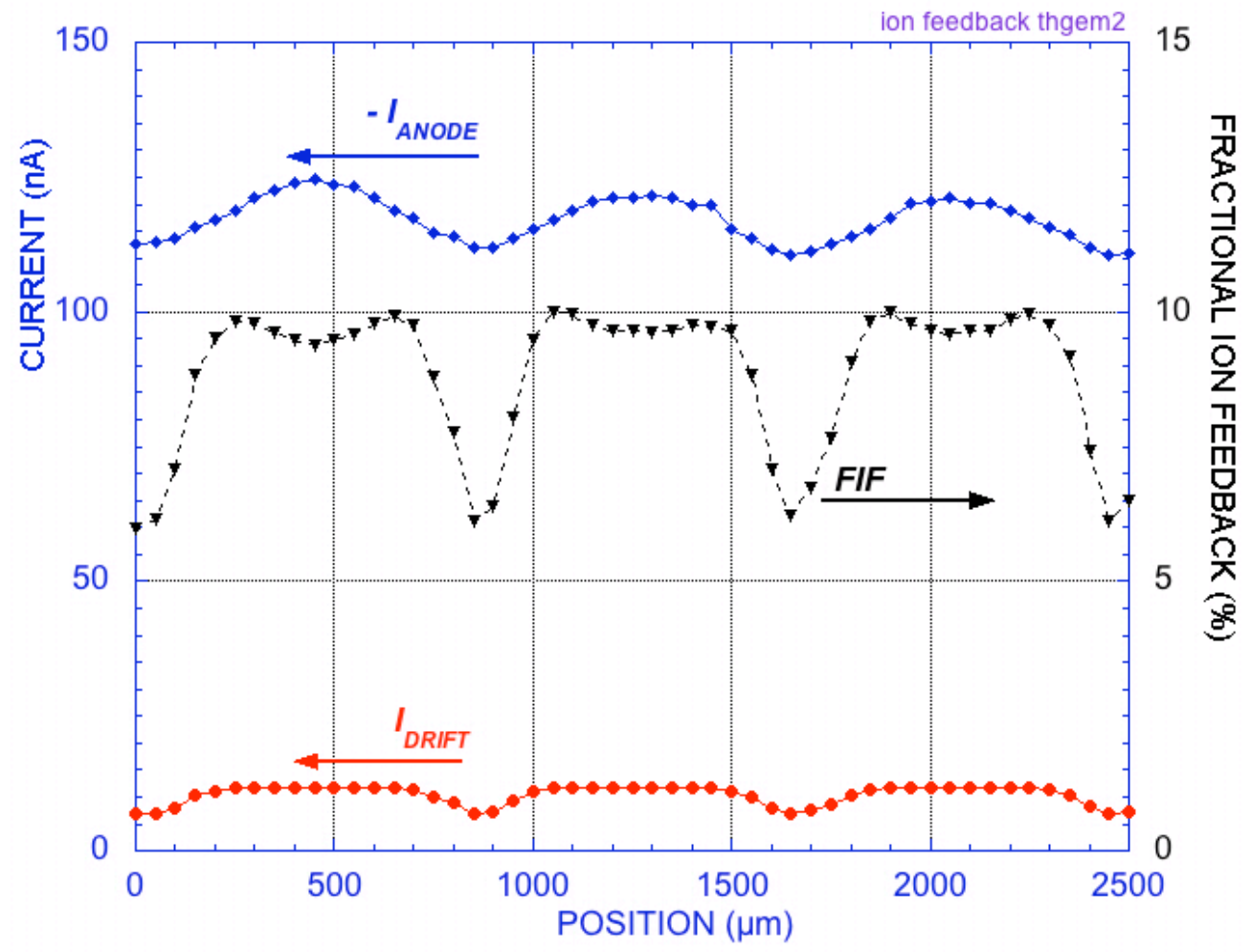

Fig. 11

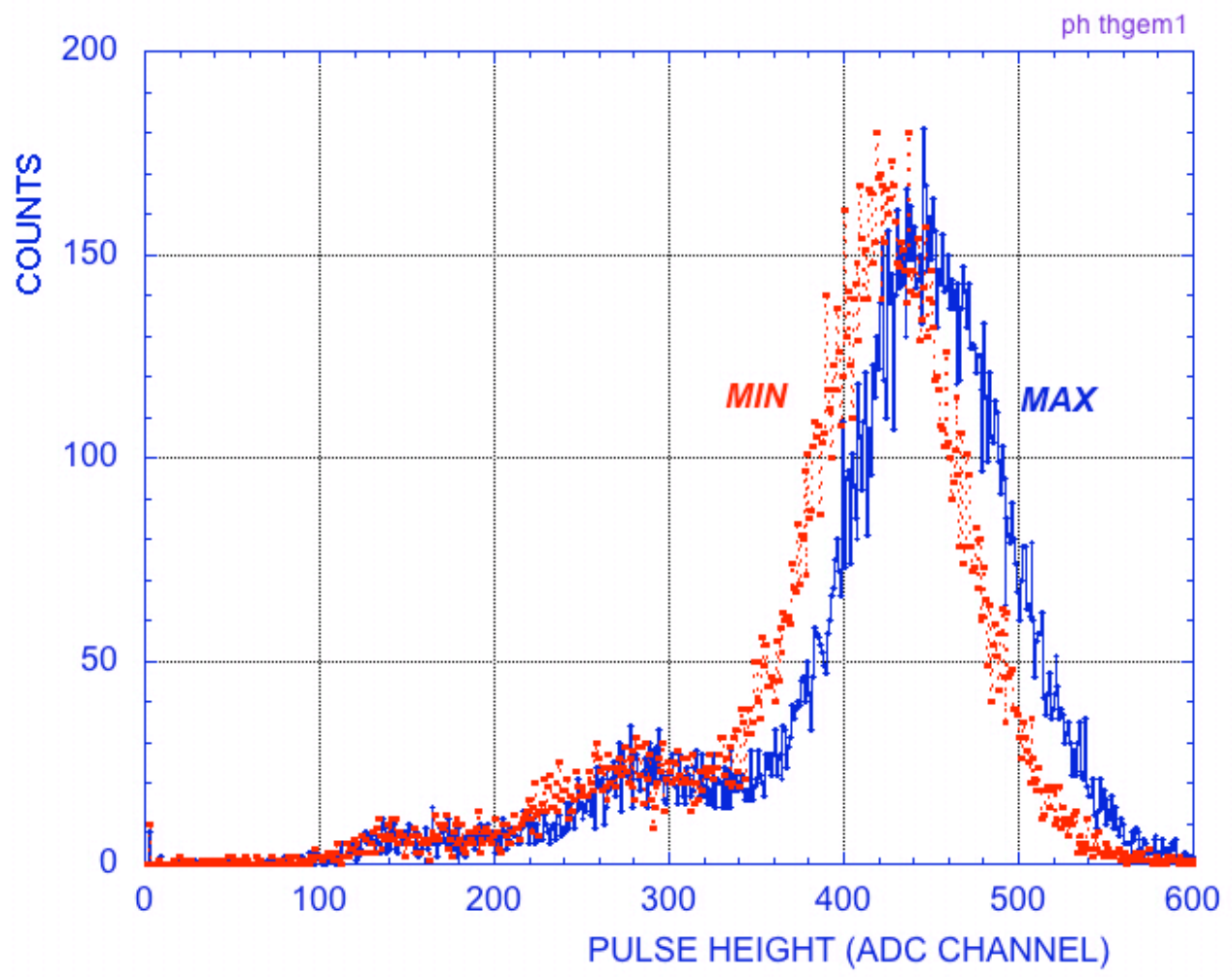

Fig. 12 